High fashion in liquid dielectrics

Simple Dielectric Liquids: Mobility, Conduction and Breakdown. By T. J. Gallagher. Pp. vi +154 . (Clarendon: Oxford; Oxford University: London, October 1975.) $£ 6.00$ net.

"BREAKDOWN in liquids could readily become a compendium of unrelated observations", wrote Whitehead in the introduction to his book on breakdown in solids. Although this pronouncement was made from the cosiness of one of those periods when that subject seemed to support an established orthodoxy, it has always held more than a grain of truth. It is the tendency to try to create an orthodoxy which has acted as an impediment to progress in all of liquids research. Arguably, such periods of stasis are little more than a state of mind among referees for scientific journals and other influential workers. The result in a notoriously difficult experimental field is a wild veering of fashion more reminiscent of haute couture than scientific progress. Everything in liquid dielectrics has been ascribed successively to ions, electrons, molecular bonds, bubbles or particles, according to vogue.

Technologically this is a very important field. In power distribution alone a small improvement in electric strength would produce enormous financial economies, and in electronics the liquid state has not begun to offer the possibilities provided by the other states of matter. It is an ideal field for $\mathrm{PhD}$ training, being generally rather too speculative for industry, yet sufficiently demanding. There has been a large volume of published work which does not seem to be matched by a corresponding degree of solid achievement. Without doubt much of this is due to the nature of the test object itself. The most critical aspect of the dielectric sample, the metal-liquid interface, is almost a completely unknown auantity. yet it determines the transfer of charge in conduction experiments and also the probability of breakdown. It must therefore be disheartening for the new researcher to find a turgid literature rife with ill-controlled experiments and glib theories.

What can one say of an author who has the temerity to attempt to survey such a field? Galagher has done as good a job as could possibly be expected. This review should rapidly become the vade-mecum for all researchers in the field, new or old. It is particularly stimulating to be able to pick out the high points in which one result condemned great chunks of the preceding literature; for example the discovery of the oxygen effect in breakdown, the e.h.d. stability theory, fast carriers in pure liquids, the statistical nature of breakdown, and so on.

If one has to find the conventional adverse criticism, it is that the author maintains his excellent disinterestedness to a fault. Having made a remark which invalidates a theory (for example, that simple equilibrium thermodynamics cannot be applied to a transient irreversible process such as breakdown) he proceeds to give it a full treatment at the expense of others which might deserve more of his limited space. A similar remark applies to some of the experimental results (for example, the use of strange techniques of statistical selection possibly inspired by vision of the Holy Grail of Intrinsic Strength). These are, however, quibbles in respect of a text which deserves to become mandatory for any serious worker in the field. The diagrams, tables, bibliography, author and subject indexes are all excellent. Readers should note that the treatment does not include the proceedings of the most recent (1975) conference, which is published by the Delft University Press. J. E. Brignell

\section{Coding for isoenzymes and haemoglobins}

Haemoglobin, Isoenzymes and Tissue Differentiation. (North-Holland $\mathrm{Re}$ search Monographs: Frontiers of Biology, Volume 42.) By C. J. Masters and R. S. Holmes. Pp. xiii +308 . (North-Holland: Amsterdam and Oxford; Elsevier Scientific: New York, 1975.) Dfl.79; $\$ 32.95$.

THE isozyme (isoenzyme) concept, initiated by Markert and Møller 16 years ago, and the accompanying techniques of zone electrophoresis and specific histochemical staining, have led to new insights in many areas of biology and biochemistry. Essentially all enzymes are capable of existing in multiple molecular forms-isozymes - whether they are encoded in different genetic loci (multiple locus isozymes) or encoded in different alleles at a locus (allelic isozymes=allozymes). This book reviews primarily those isozymes (and proteins with specific function, for example, haemoglobins) which are encoded in different but related genetic loci. Two approaches are successfully used in presenting the concepts. The first is a discussion of the molecular, genetic, and evolutionary bases of the more thoroughly documented isozyme systems. The second is the illustration of some of the ways that isozymes can be used as tools to analyse the mechanisms of genetics, physiology, development and evolution.

This is not an exhaustive com- pendium of all isozyme systems but selectively focuses on those areas of biology (cellular, developmental, genetic and evolutionary) and those enzymes, which represent the authors' areas of expertise. The results of key papers have been summarised (at times critically evaluated) and integrated into a broad contemporary biological context. The condensations of the material, the selected areas reviewed, and the thorough coverage of the earlier literature make this book a useful adjunct to the recently published four-volume proceedings of the Third International Isozyme Conference (Academic, New York) which is a comprehensive collection of original research reports covering almost all areas of biology.

The biological and experimental utility of isozymes is effectively and persuasively presented. The title is somewhat misleading, however, in that it fails to convey the evolutionary emphasis in the first third of the book. This section deals with the evolution of these multilocus isozymes (and haemoglobins), mechanisms of gene duplication, and the utilisation of these isozyme systems to establish phylogenetic relationships. A physiological rationale is often brought forward to explain the divergence in kinetic, physical and other properties of the isozymes as well as the divergence in the developmental and cellular specificity of their syntheses. There is, throughout the book, an excellent blend of evolutionary and developmental concepts. The middle third of the book analyses the differential expression of related genetic loci during development and the physiological relevance of their temporally and spatially specific syntheses.

The last third of the book is devoted to some current problems in isozymology. An interesting analysis is provided of the mechanisms involved in the regulation of isozyme levels, particularly the balanced contributions of preferential synthesis and preferential catabolism; the latter point has often been overlooked when examining isozyme repertories. In addition, a thorough analysis is made of the subcellular localisation of isozymes and the genetic and molecular basis for the specificity of the positioning of the isozymes within the cell-a topic of profound importance when considering the mechanisms of enzyme regulation. In this last section the authors indicate those areas of isozymology which will probably be the major research directions of the future. This clearly written and well organised overview of many facets of isozymes and haemoglobins should be of interest to biologists and biochemists representing a wide spectrum of research interests.

Gregory S. Whitt 